\title{
A study on anti-tumor immunity induced by gene-modified melanoma B16 cells
}

\author{
LIJUAN FANG ${ }^{1 *}$, KEMIN WANG $^{1 *}$, XIA LIU ${ }^{1,3}$, LEILEI ZHANG ${ }^{1,2}$, XINGQIAN ZHANG $^{1}$, \\ LI QIAN $^{1}$, JIAN LU ${ }^{1}$, GUANXIANG QIAN ${ }^{1}$ and SHENGFANG GE ${ }^{1,2}$ \\ ${ }^{1}$ Research Center for Human Gene Therapy, Department of Biochemistry and Molecular Biology, School of Medicine, \\ Shanghai Jiao Tong University; ${ }^{2}$ Institutes of Medical Sciences, Shanghai Jiao Tong University School of Medicine; \\ ${ }^{3}$ Department of Biochemistry, Shanghai Institute of Health Sciences, Shanghai Jiao Tong University \\ School of Medicine, Shanghai, P.R. China
}

Received August 10, 2007; Accepted October 29, 2007

\begin{abstract}
T cell-mediated cell immunity is the main antitumor immunity in which the effector $\mathrm{T}$ cells need specific antigen and costimulatory signals. One of the vaccines applied in tumor immunotherapy is the gene-modified tumor cell vaccine. One potential method to increase cell epitope density is to link the antigen with the major histcompatibility complex subunit $32 \mathrm{~m}$. Our previous research indicated that the strategy of epitope fusion gene OVA-linker- $\beta 2 \mathrm{~m}$ can promote the formation of specific compounds on the tumor cell surface in vitro. In this study, we constructed two coexpression vectors pGL3-CD80-OVA-linker- $32 \mathrm{~m}$ and pGL3-IL21-OVA-linker- $32 \mathrm{~m}$, in order to explore the cooperative action of CD80 or interleukin-21 (IL21) with the epitope fusion gene in anti-tumor immunity. Results showed that gene-modified B16 cells (B16/OVA, B16/CD80-OVA and B16/IL21-OVA) grew slower than B16 cells in vitro and in vivo, especially the B16/IL21-OVA subline, which illustrated that such gene modification decreased oncogenicity of malignant tumor cells. On the other hand, gene-modified tumor cell subline immunization can induce effective longterm anti-tumor immunity defending tumor cell attacks. IL21
\end{abstract}

Correspondence to: Dr Shengfang Ge, Research Center for Human Gene Therapy, Department of Biochemistry and Molecular Biology, School of Medicine, Shanghai Jiao Tong University, No. 280 Chongqing Road South, Shanghai 200025, P.R. China

E-mail: geshengfang@sjtu.edu.cn

${ }^{*}$ Contributed equally

Abbreviations: IL21, interleukin-21; MHC, major histocompatibility complex; PCR, polymerase chain reaction; RT-PCR, reverse transcription polymerase chain reaction; CTL, cytotoxic $\mathrm{T}$ lymphocyte; NK cell, natural killer cell; ELISA, enzyme linked immunosorbent assay; ICAM-I, intercellular adhesion molecule-I; AICD, activation-induced cell death; PBMC, peripheral blood mononuclear cells; HBSS, hank's balanced salt solution

Key words: melanoma, epitope fusion gene, CD80, interleukin-21, immunogene therapy played a more cooperative role with the OVA-linker- $32 \mathrm{~m}$ than CD80 in this study. This strategy might lay foundations for the research of a new type of tumor vaccine.

\section{Introduction}

Recent research indicates that the possible mechanisms that allow tumor cells to escape from immune system attacks are tumor antigen modulation, weak immunogenicity, the lowexpression or lack of MHC molecule, the low expression of a costimulatory molecule and lack of local cytokines. Many factors eventually cause the local immune-suppressive state of tumors and the immune effector cells cannot stimulate the effective anti-tumor immune response. On the other hand, a tumor can also edit the immune system, which means that long-term, existent and chronic stimulation of a tumor leads to immune system exhaustion even tumor-specific $\mathrm{T}$ cell clonal deletion (1). Therefore, the main purpose of tumor immunotherapy at present is to try to reverse the local immune-suppressive state, enhance the original weak tumor immunogenicity and improve the quantity of local activated immune effector cells so as to stimulate a strong anti-tumor immunity response.

One of the vaccines applied in tumor immunotherapy is the gene-modified tumor cell vaccine, which can enhance tumor cell immunogenicity and degrade tumorigenesis through gene modification. One potential method is to link a specific epitope with $\beta 2$ microglobulin $(\beta 2 \mathrm{~m})$ molecules in order to enhance tumor cell epitope density. $\beta 2 \mathrm{~m}$ is irrelevant with the antigenicity of the MHC-I molecule, though it is necessary for the correct expression and normal function of the MHC-I heavy chain (2,3). We previously constructed an OVA-linker- $\beta 2 \mathrm{~m}$ fusion vector, which served as an immunogen and successfully induced the specific CTL cells. A previous study has verified that the OVA-linker- $32 \mathrm{~m}$ fusion gene can promote the formation of a specific compound on the cell surface which accordingly enhances the tumor cells' immunogenicity $(4,5)$.

Based on previous work, we wished to investigate whether the method of fusion gene can induce effective antitumor immunity in the weak immunogenicity tumor model. The weak immunogenicity of tumor cells is partly due to the 
low expression of costimulatory molecules. Without a costimulatory signal, $\mathrm{T}$ cells cannot be activated thoroughly and can even be in an anergy condition $(6,7)$. The costimulatory molecule, CD80, plays an important role in the process of $\mathrm{T}$ cell activation (8). In addition, the lack of cytokines is one of the reasons for the tumor's escape from the immune system. IL21 is a new member of the IL-2 family, mainly produced by the activated $\mathrm{CD} 4^{+} \mathrm{T}$ cells. It has extensive biological activities, especially in enhancing the functions of the effector T cell and activated NK cell, which can effectively strengthen the innate immunity and specific immunity and promote the tumor-bearing animal to produce anti-tumor immunity $(9,10)$. In contrast with IL2 treatment, which induces activation-induced cell death (AICD), IL21 sustains the number of $\mathrm{CD}^{+} \mathrm{T}$ cells as a result of increased survival (11). So we constructed two new vectors, which respectively coexpress the OVA-linker- $\$ 2 \mathrm{~m}$ fusion protein and CD80, or IL21, in order to study whether combining gene-modification of tumor cells can induce a more intense anti-tumor immunological effect.

\section{Materials and methods}

Mice and cell lines. Female C57BL/6 mice, 6-8 weeks of age, were treated according to the NIH Guidelines for Animal Care in the Experimental Animal Center of Shanghai Jiao Tong University School of Medicine. Melanoma B16 cells of mice were obtained from the cell bank of Academia Sinica and were maintained in RPMI-1640 medium supplemented with $10 \%$ fetal bovine serum (Hyclone), $2 \mathrm{M}$ pyruvate, $50 \mu \mathrm{M}$ 2-mercaptoethanol, penicillin $(200 \mathrm{U} / \mathrm{ml})$ and streptomycin $(200 \mu \mathrm{g} / \mathrm{ml})$ at $37^{\circ} \mathrm{C}$ in a humidified atmosphere containing $5 \% \mathrm{CO}_{2}$. For gene-modified cells, Geneticin $(500 \mu \mathrm{g} / \mathrm{ml})$ (Invitrogen) was added to the medium.

Expression vector construct. The following plasmid vectors were constructed: i) pCDNA3-OVA-linker- $\$ 2 \mathrm{~m}$, ii) pGL3CD80-OVA-linker-32m, iii) pGL3-IL21-OVA-linker-ß2m.

The plasmid pCDNA3-OVA-linker- $32 \mathrm{~m}$ was constructed (4). The epitope $\mathrm{OVA}_{256-264}$ is the sequence of nine peptides which has high affinity with $\mathrm{H}-2 \mathrm{~K}^{\mathrm{b}}$. The epitope sequence is linked with human $\beta 2 \mathrm{~m}$ by a short linker sequence mainly made up of glycine and serine (GGGSGGGGS). To construct the plasmid pGL3-CD80-OVA-linker- $32 \mathrm{~m}$, the plasmid pCDNA3-OVA-linker-B2m and pGL3-CD80-P1A were digested with BglII and SmaI enzymes, the 450 bp BglII-SmaI OVA fusion gene was cloned into the same enzyme digested plasmid backbone pGL3-CD80. The resulting vector was named pGL3-CD80-OVA-linker- $\$ 2 \mathrm{~m}$. To construct a plasmid pGL3-IL21-OVA-linker-ß2m, we cloned the mouse IL21 gene from a mouse peripheral blood mononuclear (PBMC) with a $5^{\prime}$ primer ccc aag ctt ctc ctg gag act cag ttc tgg and a 3' primer tgc tct aga tca gga atc ttc ggg tcc ta (9). The upstream primer incorporated a HindIII site at the $5^{\prime}$ end and the downstream primer incorporated an XbaI site. Then the PCR product of IL21 was digested with HindIII and XbaI and cloned into the same enzyme-digested vector pGL3promoter. After constructing the plasmid pGL3-IL21, the 450 bp BglII-SmaI OVA fusion gene was cloned into the BglII and SmaI digested pGL3-IL21 vector similarly. The final vector was named pGL3-IL21-OVA-linker-ß2m. All of these constructs were confirmed by the enzyme digestion and sequence analyses.

Cell transfection. Tumor cells were transfected with plasmid DNA using liposome Lipofectamin 2000 according to the manufacturer's manual (Invitrogen). Briefly, B16 cells were plated at a concentration of $2 \times 10^{5}$ cells per well in a $2 \mathrm{ml}$ medium in a 6 -well plate before transfection and grew to $60-70 \%$ confluency. As the two new plasmids have no selective gene Neo, the plasmid pSV40-neo is needed for cotransfection. The plasmid/liposome complexes were prepared $30 \mathrm{~min}$ before transfection. Cells were incubated with plasmid/liposome complexes for $6 \mathrm{~h}$. Then the medium was replaced with a fresh culture medium. At the same time, the transfection efficiency was determined by simultaneous transfection of plasmid pCDNA3-EGFP. While selecting stable transgene clones, the tumor cells were grown in $500 \mu \mathrm{g} /$ $\mathrm{ml}$ Geneticin containing medium $48 \mathrm{~h}$ after transfection and continued to select monoclones by limiting dilution in 96-well plates. The expression of the transgene was confirmed by the reverse transcription PCR. Tumor cell surface expression of CD80 was determined by flow cytometry. Cytokine IL21 expression was detected by an enzyme-linked immunosorbent assay (ELISA).

$R T-P C R$ reaction. The total RNA of either wild-type B16 cells or the stable transfected tumor cells was isolated by using Trizol agent (Invitrogen). The RNA samples were reverse transcribed according to the manufacturer's instructions using reverse transcriptase (Invitrogen), random hexamers and dNTP. The synthesized cDNAs were amplified by PCR (both 30 cycles for OVA, CD80, IL21 and B-actin) with Taq DNA polymerase in the presence of both $\mathrm{dNTP}$ and an appropriate pair of primers. Table I shows the sense and anti-sense primers used.

Measurement of CD80 on transfected B16 tumor cells. The CD80 expression on the cell surface was determined by flow cytometry. All labeling steps were carried out at $4^{\circ} \mathrm{C}$. Wildtype tumor cells and gene-modified cells were collected and incubated for 30 min with a FITC-anti-mouse CD80 antibody (eBioscience), then washed twice and suspended in fixing solution. Stained cells were analyzed by FACS.

IL21 expression assays. The cytokine IL21 expression was analyzed by an enzyme-linked immunosorbent assay (ELISA) according to the manufacturer's protocol (R\&D System). Briefly, a 96-well plate was incubated with a goat anti-mouse IL21 antibody and blocked with blocking buffer. After washing, a standardized IL21 solution and 24 and 48 h cell culture supernatants were then added to the wells. Following $2 \mathrm{~h}$ of incubation, the plate was washed three times. Biotinlabeled detection antibody and HRP-conjugated antibody were added respectively. After another hour of incubation and washing, the substrate solution was added and the plate was read at $450 \mathrm{~nm}$.

Tumorigenicity of the gene-modified tumor cells. Confluent cultures of gene-modified and wild-type tumor cells were 
Table I. The sense and anti-sense primers.

OVA-linker- $\beta 2 \mathrm{~m}$

sense

OVA-linker- $32 \mathrm{~m}$

anti-sense

CD80 sense

CD80 anti-sense

IL21 sense

IL21 anti-sense

ß-actin sense

B-actin anti-sense
5' CAG CAT ATG TCC ATA ATC AAC TTT GAA AAA CTC GGA AGG
AGG ATC CGA GGT GGC AGC ATC CAG CGT ACT CCA AAG-3'

5' CAACTCGAGCATGTCTCGATCCCAC-3'

5' CAAAGCATCTGAAACCATGGCT-3'

5' CTAAAGGAAGACGGTC-3'

5' CCCAAGCTTCTCCTGGAGACTCAGTTCTGG-3'

5' TGCTCTAGATCAGGAATCTTCGGGTCCTA-3'

5' TCGACAACGGCTCCGGCAT-3'

5' CACAATCCACCAGCCAGCCTCA-3'
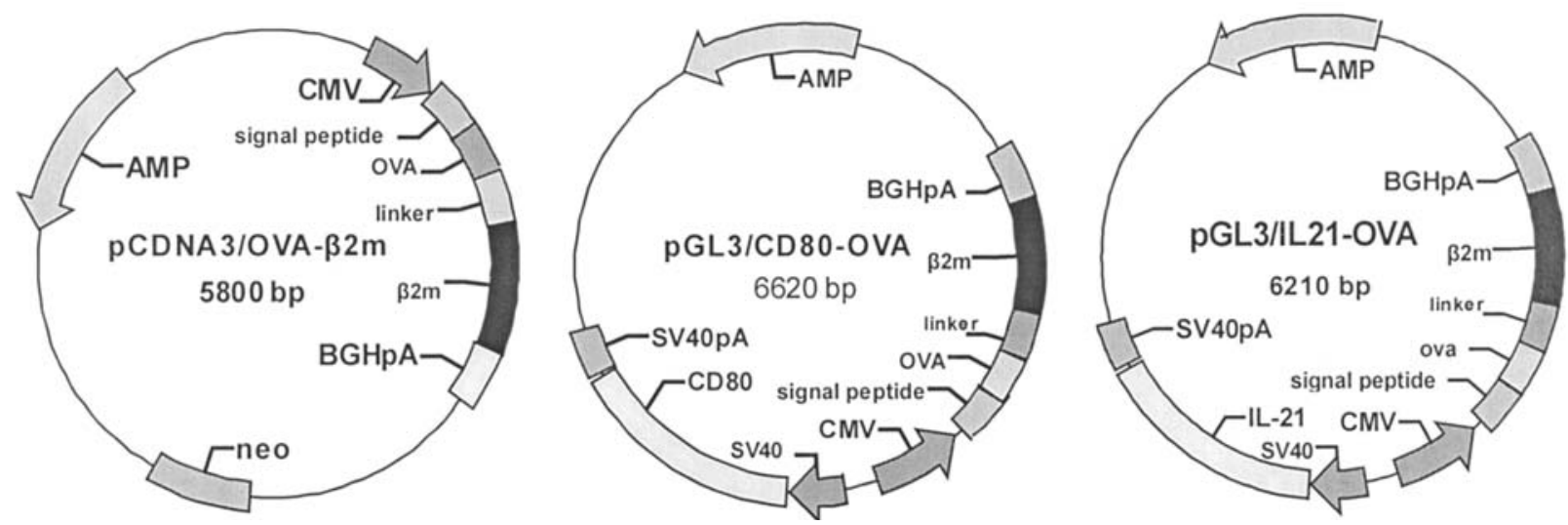

Figure 1. A schematic diagram describing the different OVA-linker- $32 \mathrm{~m}$ expression vectors. pCDNA3-OVA- $32 \mathrm{~m}$ : CMV promoter driving the expression of the epitope fusion gene, in which the encoding gene of antigen epitope OVA is linked with the human $\$ 2$ microglobulin gene by a short linker sequence (GGGSGGGGS). PGL3-CD80-OVA-B2m: The original Neo gene was replaced by the mouse CD80 gene. The SV40 promoter is driving the expression of mouse CD80, while the CMV promoter is driving the expression of the OVA-linker-32m fusion protein. PGL3-IL21-OVA- $32 \mathrm{~m}$ : The original Neo gene was replaced by the mouse IL21 gene. The SV40 promoter is driving the expression of mouse IL21, while the CMV promoter is driving the expression of the OVA-linker- $\beta 2 \mathrm{~m}$ fusion protein.

harvested and inoculated subcutaneously in the right flank of syngenic C57BL/6 mice (six animals per group) with $1 \times 10^{5}$ viable tumor cells in $0.1 \mathrm{ml}$ of HBSS. Tumors were measured every 3 days with calipers once the tumors became palpable. The tumor volume was calculated using the following formula: length $\mathrm{x}$ width $2 / 2$.

Immunization with genetically modified tumor cells. C57BL/6 mice were immunized s.c. with either $1 \times 10^{6}$ irradiated wildtype B16 cells or gene-modified B16 cells, which were B16/ OVA, B16/CD80-OVA and B16/IL21-OVA. The immunization procedure was repeated 7 days later. One week after the final immunization, mice of four groups were challenged s.c. with $10^{5}$ viable tumor cells. Then, the animals were monitored for more than 60 days and the tumor load was measured in each group.

\section{Results}

Assay of fusion gene expression in B16 cells through RT-PCR. After transfection and Geneticin selection, the stable transfectants were referred to as B16/OVA, B16/CD80-OVA and B16/IL21-OVA, respectively. RT-PCR results showed that transfectants had significantly up-regulated the expression of OVA-linker-B2m, CD80 and IL21 mRNA (Fig. 2). In contrast, B16 cells showed no expression of these genes.

The expression of the CD80 molecule on the surface of $G 418$ selected. The wild-type B16 tumor cells and the stable transfected cell lines B16/CD80-OVA were evaluated by flow cytometry for the expression of CD80. The data showed that B16 cells were almost negative for CD80 expression while B 16/CD80-OVA cells were $70.62 \%$ positive for CD80 expression 2-3 weeks following transfection and subsequent culture in a medium containing G418 as shown in Fig. 3.

ELISA analysis of IL21 secreted by the G418 selected cells. Culture supernatants from different cell lines (B16, B16/OVA and B16/IL21-OVA) were collected and assayed for IL21 by ELISA. Culture supernatants from B16, B16/OVA contained hardly detectable levels of IL21, while supernatants from stable transfected B16/IL21-OVA contained high levels of IL21 $48 \mathrm{~h}$ after culture in the medium (Fig. 4). There is an obvious difference between B16, B16/OVA and B16/IL21-OVA. 


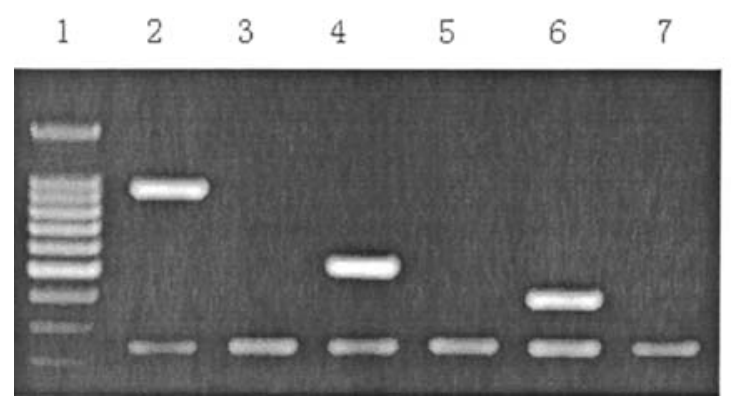

Figure 2. Assay of fusion gene expression in B16 cells through RT-PCR. Lane 1 is the 100 bp DNA ladder marker. Lane 2 is the 920 bp RT-PCR result of mouse CD80 gene from B16/CD80-OVA cell total RNA, while CD80 expression is almost negative in B16 RNA in lane 3. Similarly, lane 4 is the 508 bp RT-PCR result of the mouse IL21 gene from B16/IL21-OVA cell total RNA and its expression is negative in the B16 control group in lane 5. Lane 6 is the $450 \mathrm{bp} \mathrm{RT-PCR} \mathrm{result} \mathrm{of} \mathrm{the} \mathrm{OVA} \mathrm{fusion} \mathrm{gene} \mathrm{from}$ B16/OVA RNA and there is no expression in the B16 control group in lane 7. All the tumor cells show 239 bp B-actin gene expression.

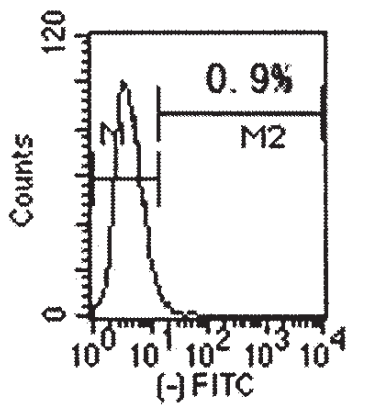

B16

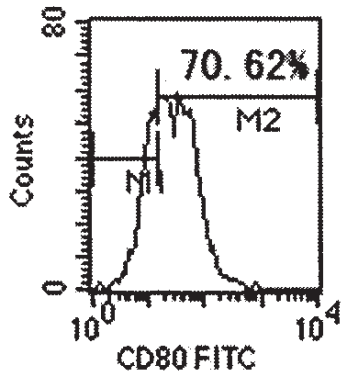

$B 16 / C D 80$
Figure 3. The expression of the CD80 molecule on the surface of G418 selected transfectants. After transfection and subsequent culture in a medium containing G418, the cultured B16 and B16/CD80 cells were collected and the expression of CD80 was detected by FACS. B16/CD80 cell surface shows a high level of expression of CD80 ( 70.62\%), while it is almost negative on the B16 cell surface. The result is relevant with the RT-PCR analysis.

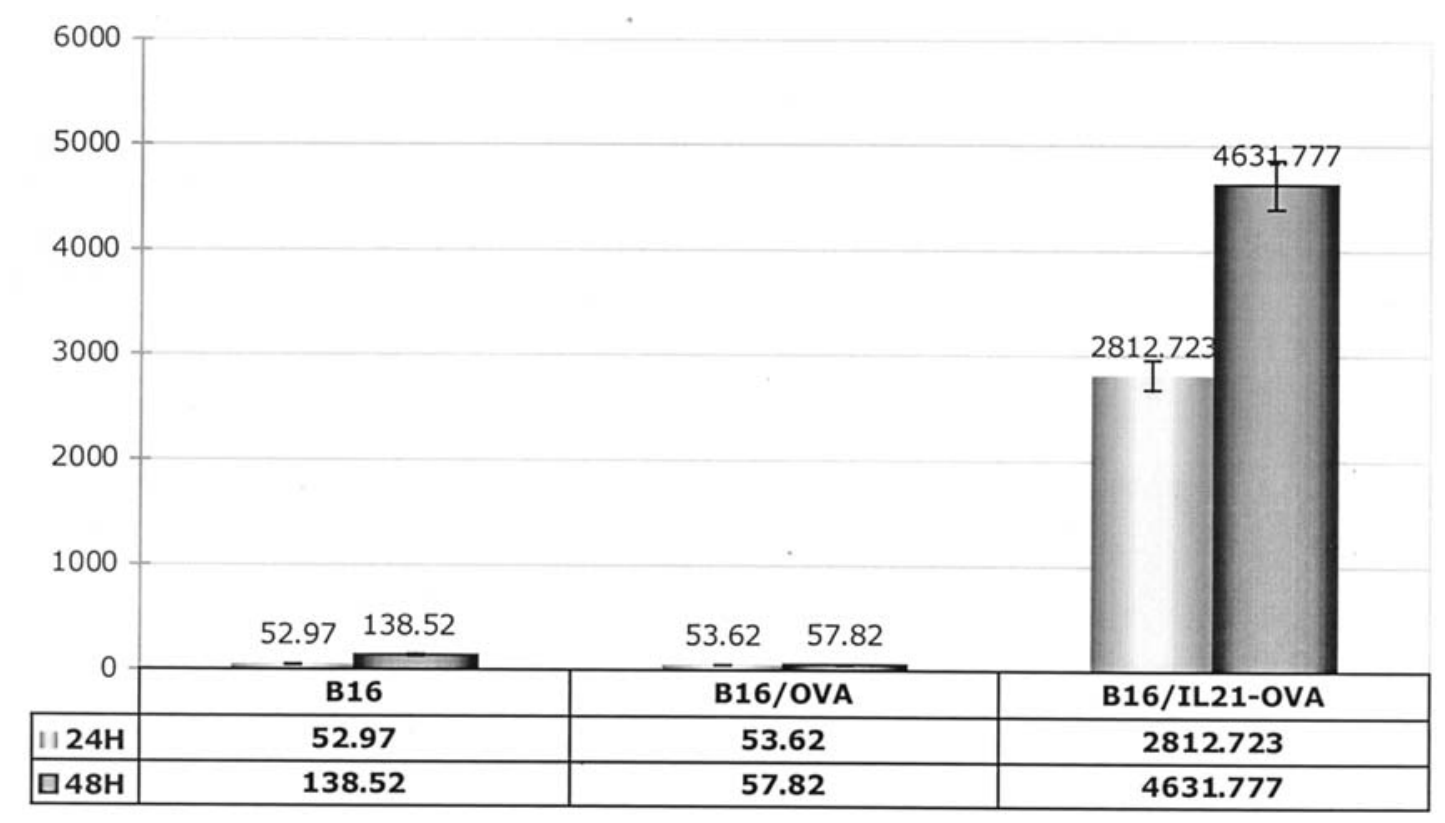

Figure 4. ELISA analysis of IL21 secreted by the G418 selected cells. Twenty-four and 48 h culture supernatants from B16, B16/OVA and B16/IL21-OVA were collected and analyzed by ELISA, respectively. B16 and B16/OVA cell culture supernatants showed hardly detectable levels of IL21. However, the B16/IL21-OVA cell secretes high levels of IL21, that is $2812.72 \mathrm{pg} / \mathrm{ml}$ and $4631.77 \mathrm{pg} / \mathrm{ml}$ after 24 and $48 \mathrm{~h}$ of culture, respectively.

Cell growth curves of gene-modified B16 cells. An in vitro cell proliferation assay showed that the growth tendency curves of stable transfectants were different from the control B16 cells. B16/OVA, B16/CD80-OVA and B16/IL21-OVA sublines grew significantly slower than the B16 cells $(\mathrm{P}<0.05)$, especially the B16/IL21-OVA cell lines (Fig. 5).

Assay of the tumorigenicity of gene-modified B16 cells. C57BL/6 mice (six mice in each group) were injected subcutaneously with $1 \times 10^{5}$ wild-type B 16 cells or the different stable B16 sublines. Tumors were monitored every 3 days once the tumor became palpable. Results suggested that the antigen epitope fused with the $32 \mathrm{~m}$ strategy resulted in decreased tumorigenicity and tumors derived from epitope and IL21 expressing B16 sublines grew significantly slower than the wild-type B16 cell group $(\mathrm{P}<0.05)$. However, there was no obvious difference between the B16/CD80-OVA subline group and the B16 cell group ( $\mathrm{P}>0.05)$ (Table II).

The anti-tumor protective immunity induced by gene-modified B16 immunization. We then explored the possibility that immunization with gene-modified B16 cells might afford protection against tumor cell attacks. Four groups of C57BL/6 mice (five mice in each group) were immunized with irradiated B16, B16/OVA, B16/CD80-OVA or B16/IL21OVA cells, respectively. Following immunization, mice were challenged s.c. with different transfected B 16 cells and monitored for tumor load. Mice immunized with B16 cells rapidly developed aggressive tumors and showed no evidence of protection from tumor challenge. On the other hand, mice 
Table II. Average tumor volume of each group (unit: $\mathrm{cm}^{3}$ ).

\begin{tabular}{lcccc}
\hline Days & 10 & 14 & 17 & 21 \\
\hline B16 & $0.220 \pm 0.008$ & $0.222 \pm 0.032$ & $0.596 \pm 0.027$ & $1.697 \pm 0.342$ \\
B16/OVA & $0.048 \pm 0.013$ & $0.109 \pm 0.047$ & $0.381 \pm 0.027$ & $0.852 \pm 0.670$ \\
B16/CD80-OVA & $0.091 \pm 0.033$ & $0.165 \pm 0.101$ & $0.454 \pm 0.290$ & $1.206 \pm 0.702$ \\
B16/IL21-OVA & $0.018 \pm 0.010$ & $0.081 \pm 0.093$ & $0.266 \pm 0.084$ & $0.817 \pm 0.295$ \\
\hline
\end{tabular}

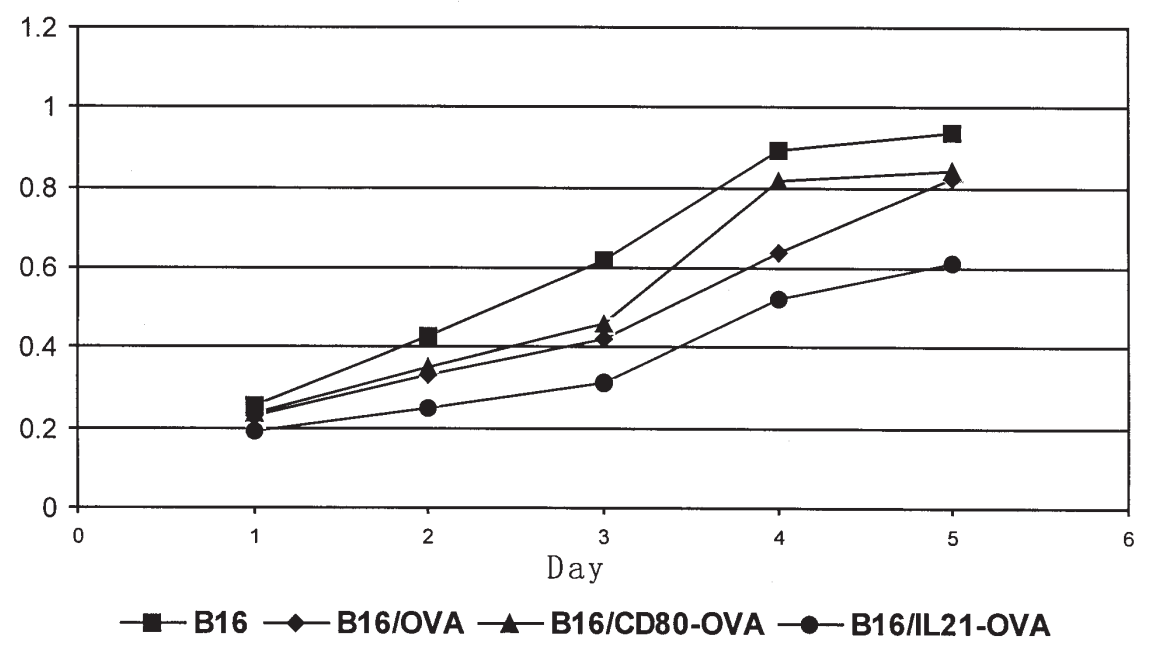

Figure 5. Cell growth curves of gene-modified B16 cells. Gene-modified B16 cells and B16 cells were plated at the concentration of 1,000 cells per well in a $100 \mu 1$ medium in 96-well plates, respectively. Twenty $\mu 1$ of MTS was added into each well from days 1 to 7 . After $2 \mathrm{~h}$ of culture, the results were read under a $490 \mathrm{~nm}$ wave length. The gene-modified B16 cells grew significantly slower than the control B16 cells. B16/IL21-OVA cells grew the slowest.

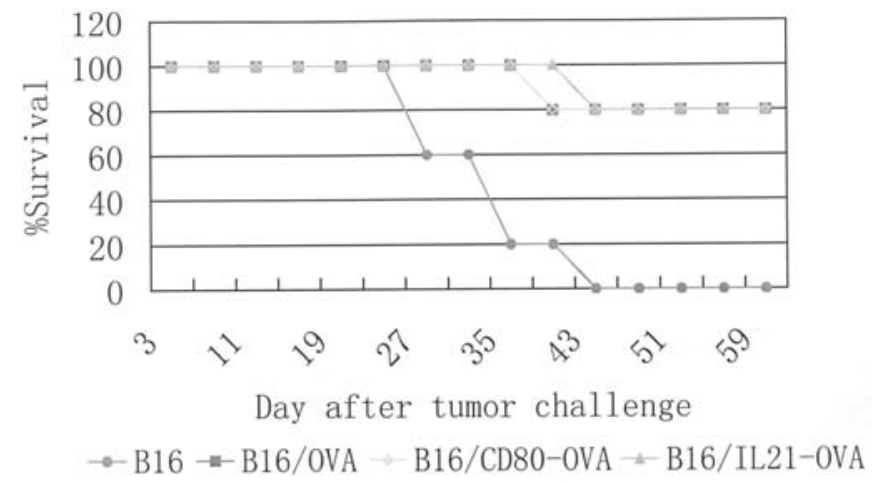

Figure 6 . The anti-tumor protective immunity induced by gene-modified B16 immunization. Four groups of $\mathrm{C} 57 \mathrm{BL} / 6$ mice were immunized with $1 \times 10^{6}$ irradiated different gene-modified B16 cells and control B16 cells, respectively. After immunization, each mouse was challenged with $1 \times 10^{5}$ viable transfected B16 cells and control B16 cells. By day 42, five mice in the B16 group all died. In contrast, only one mouse in the other three groups died, while the remaining four mice in each group were in a tumor-free condition for more than 60 days.

immunized with gene-modified B16 cells showed strong protection and tumors grew much less aggressively. By day 42, mice immunized with B16 cells all died, while $80 \%$ of mice in each of the other three groups were still alive under a tumor-free condition for more than 60 days. By day 64, the mice that survived were challenged again with tumor cells and monitored for tumor load. Notably, only $1 / 4$ of the mice in each group developed tumors and grew much less aggressive than before (data not shown). This suggests that animals immunized with gene-modified B16 cells show strong protection against tumor attack and gain a long-term immune response.

\section{Discussion}

$\mathrm{T}$ cell-mediated cell immunity is one of the anti-tumor immunity mechanisms. The activation of $\mathrm{T}$ cells needs two signals: one is the signal provided by the $\mathrm{T}$ cell receptor and MHC-peptide compound recognition; another is the costimulatory signal provided by the costimulatory molecules. The MHC/peptide compound density on the antigen presenting cell (APC) surface directly determines the intensity of a T cell response (12). The expression of MHC on melanoma cell surface is very low, resulting in a weak first signal and is unable to induce thorough the activation of T cells (13-15).

It has been reported that a potential method to enhance cell epitope density is to link the specific epitope with the MHC-I heavy chain, or to link an antigen epitope with an exogenous $\beta 2$ microglobulin molecule (16). The construction strategy of the ova-linker- $\beta 2 \mathrm{~m}$ fusion gene vector is verified to facilitate the formation and stability of the MHC/peptide 
compound, which served as an effective immunogen and successfully induced the specific CTL cells. In addition, a mechanism that prevents antigen-specific $\mathrm{T}$ cells from activation and causing local immunity tolerance in the tumor microenvironment is the low expression of the costimulatory molecule on the tumor cell surface $(17,18)$. CD80, as a costimulatory molecule, plays an important role in $\mathrm{T}$ cell activation (8). The role of cytokines in $\mathrm{T}$ cell activation is also vital. It has been named the 'third signal' (19). Previous research indicated that the microenvironment of cytokines is an important link in determining the differentiation of $\mathrm{T}$ cells and the subsequent characteristics of the immune response (20). That is to say, we can co-transfect CD80 and some cytokines in order to stabilize the cooperation of the microenvironment of $\mathrm{T}$ cells in the anti-tumor immunity responses.

Interleukin (IL), especially the IL-2 family plays an important role in anti-tumor immunity or adjunctive therapy. IL21 is noted for its strong immunological regulation function. We constructed two coexpression vectors, which coexpress the OVA fusion protein and CD80, or OVA fusion protein and IL21 respectively, in order to gain an intense anti-tumor effect. The data of the in vitro proliferation assay indicated that gene-modified B16 cells B16/OVA, B16/CD80-OVA and B16/IL21-OVA grew significantly slower than wild-type B16 cells, especially the B16/IL21OVA cells $(\mathrm{P}<0.05)$. The possibility is that cytokine IL21 autocrined by B16/IL21-OVA cells directly inhibited the tumor cell growth, which is also verified by the result of ELISA. Oncogenicity tests suggested that the B16/OVA and B16/IL21-OVA sublines grew slower in vivo than B16 cells. However, there was no obvious growth difference between the B16/CD80-OVA sublines and B16 cells $(\mathrm{P}>0.05)$. A report by Chen et al (21) pointed out that the immunogenicity of tumor cells decides the costimulation effect of CD80 in T cell-mediated anti-tumor immunity. That is, oncogenicity of immunogenic tumor cells (P815, EL4) degraded when transfected with CD80 gene. However, transfection with the CD80 gene did not influence the oncogenicity of nonimmunogenic tumor cells, such as B16 cells. It was also suggested that the introduction of the CD80 gene can enhance the oncogenicity of B16 cells, though the effect of CD80 was not as strong as it was on immunogenic tumor cells. This phenomenon might be relevant with the expression levels of ICAM-I or MHC molecules on the tumor cell surface (22-24). This may be one of the reasons that illustrate why the coexpression of the CD80 molecule did not significantly degrade the oncogenicity of B16 cells in vivo.

In a further exploration of the immunoprotection assay, we challenged the experimental group of mice with the same modified B16 cells instead of the wild-type B16 cell after immunization. The results showed that, compared with the control group, gene-modified B16 cell vaccines can induce strong protection against tumor cell attacks and also gain a long-term immunity response, which illustrated the activation of the in vivo immune system and the strengthening of the function of effector immune cells while the control group cannot induce an effective immune response. A gene-modified tumor vaccine might activate an adaptive immunity mechanism that gains long-term immunity. It was reported by Guo et al $(25,26)$ that gene-modified tumor cell vaccines can elicit tumor-specific anti-tumor immunity and the activated effector CTLs can kill not only gene-modified tumor cells, but also parental tumor cells because of the in vivo immune system activation and gene-modified tumor cell growth retardation. Apoptosis can release more specific tumor antigen in order to induce effective immunity. It can be said that immunization of gene-modified B16 cells elicit such a strong tumor-specific immunity that it can also protect mice from parental B16 cell attacks through direct CTLs or indirect cytokine function.

Based on the above, we can conclude that the epitope fusion gene OVA-linker- $\$ 2 \mathrm{~m}$ modified melanoma cells grow slower than wild-type melanoma cells both in vitro and in vivo and its cell immunization induces effective anti-tumor immunity. Enhancement of the second signal provided by coexpression of CD80 does not significantly degrade the melanoma cell oncogenicity, although its immunization is affected. However, by increasing the amount of cytokine IL21 in a tumor micro-enviroment does strengthen the epitope fusion protein's effect on melanoma cells in vitro and in vivo. These results contribute to the research and development of new types of tumor vaccines which have latent applied values.

\section{Acknowledgements}

This work was supported by The National Key Program for Basic Research of China (2004CB518804), The Science and Technology Commission of Shanghai (04JC14041 and 07JC14034) and The Fund for the cooperation of basic and applied research supported by Renji Hospital (ZD0702).

\section{References}

1. Chan L, Hardwick NR, Guinn B-a, et al: An immune edited tumour versus a tumour edited immune system: prospects for immune therapy of acute myeloid leukaemia. Cancer Immunol Immunother 55: 1017-1024, 2006.

2. Kozlowski S, Takeshita T, Boehncke WH, et al: Excess beta 2 microglobulin promoting functional peptide association with purified soluble class I MHC molecules. Nature 349: 74-77, 1991.

3. Vitiello A, Potter TA and Sherman LA: The role of beta 2microglobulin in peptide binding by class I molecules. Science 250: 1423-1426, 1990.

4. Qian L and Qian GX: In vivo CTL immunity can be elicited by OVA-linker-beta $2 \mathrm{~m}$ fusion protein. Sheng wu hua xue yu sheng wu wu li xue bao Acta biochimica et biophysica Sinica 34: 547-552, 2002.

5. Uger RA and Barber BH: Creating CTL targets with epitopelinked beta 2-microglobulin constructs. J Immunol 160: 1598-1605, 1998.

6. Schwartz RH: T cell anergy. Annu Rev Immunol 21: 305-334, 2003.

7. Carreno BM, Carter LL and Collins M: Therapeutic opportunities in the B7/CD28 family of ligands and receptors. Curr Opin Pharmacol 5: 424-430, 2005.

8. Liu X, Zhang LL, Zhang XQ, et al: The effect of co-expression costimulatory molecule CD80 on uptake of antigen peptide-MHC class I-GFP complex by specific T cells. Int J Oncol 30: 1389-1396, 2007

9. Parrish-Novak J, Dillon SR, Nelson A, et al: Interleukin 21 and its receptor are involved in NK cell expansion and regulation of lymphocyte function. Nature 408: 57-63, 2000.

10. Kasaian MT, Whitters MJ, Carter LL, et al: IL-21 limits NK cell responses and promotes antigen-specific $\mathrm{T}$ cell activation: a mediator of the transition from innate to adaptive immunity. Immunity 16: 559-569, 2002.

11. Moroz A, Eppolito C, Li Q, Tao J, Clegg CH and Shrikant PA IL-21 enhances and sustains $\mathrm{CD} 8^{+} \mathrm{T}$ cell responses to achieve durable tumor immunity: comparative evaluation of IL-2, IL-15, and IL-21. J Immunol 173: 900-909, 2004. 
12. Engelhard VH: Structure of peptides associated with class I and class II MHC molecules. Annu Rev Immunol 12: 181-207, 1994.

13. Lurquin C, Van Pel A, Mariame B, et al: Structure of the gene of tumor transplantation antigen P91A: the mutated exon encodes a peptide recognized with Ld by cytolytic T cells. Cell 58: 293-303, 1989.

14. Baskar S, Clements VK, Glimcher LH, Nabavi N and Ostrand-Rosenberg S: Rejection of MHC class II-transfected tumor cells requires induction of tumor-encoded B7-1 and/or B7-2 costimulatory molecules. J Immunol 156: 3821-3827, 1996.

15. van Seventer GA, Newman W, Shimizu Y, et al: Analysis of T cell stimulation by superantigen plus major histocompatibility complex class II molecules or by CD3 monoclonal antibody: costimulation by purified adhesion ligands VCAM-1, ICAM-1, but not ELAM-1. J Exp Med 174: 901-913, 1991.

16. Rock KL, Fleischacker C and Gamble S: Peptide-priming of cytolytic $\mathrm{T}$ cell immunity in vivo using beta 2 -microglobulin as an adjuvant. J Immunol 150: 1244-1252, 1993.

17. Ochsenbein AF, Sierro S, Odermatt B, et al: Roles of tumour localization, second signals and cross priming in cytotoxic T-cell induction. Nature 411: 1058-1064, 2001.

18. Zippelius A, Batard P, Rubio-Godoy V, et al: Effector function of human tumor-specific CD8 T cells in melanoma lesions: a state of local functional tolerance. Cancer Res 64: 2865-2873, 2004.
19. Langowski JL, Zhang X, Wu L, et al: IL-23 promotes tumour incidence and growth. Nature 442: 461-465, 2006.

20. Coutelier JP, Van Broeck J and Wolf SF: Interleukin-12 gene expression after viral infection in the mouse. J Virol 69: 1955-1958, 1995.

21. Chen L, McGowan P, Ashe S, Johnston J, Li Y, Hellstrom I and Hellstrom KE: Tumor immunogenicity determines the effect of B7 costimulation on T cell-mediated tumor immunity. J Exp Med 179: 523-532, 1994

22. Guckel B, Lindauer M, Rudy W, et al: CD80-transfected human breast and ovarian tumor cell lines: improved immunogenicity and induction of cytolytic $\mathrm{CD}^{+} \mathrm{T}$ lymphocytes. Cytokines $\mathrm{Mol}$ Ther 1: 211-221, 1995.

23. Chen L, Linsley PS and Hellstrom KE: Costimulation of T cells for tumor immunity. Immunol Today 14: 483-486, 1993.

24. Cavallo F, Martin-Fontecha A, Bellone M, et al: Co-expression of B7-1 and ICAM-1 on tumors is required for rejection and the establishment of a memory response. Eur J Immunol 25: 1154-1162, 1995.

25. Liu Y, Wang H, Zhao J, Ma J, et al: Enhancement of immunogenicity of tumor cells by cotransfection with genes encoding antisense insulin-like growth factor-1 and B7.1 molecules. Cancer Gene Ther 7: 456-465, 2000.

26. Guo YJ, Che XY, Shen F, et al: Effective tumor vaccines generated by in vitro modification of tumor cells with cytokines and bispecific monoclonal antibodies. Nat Med 3: 451-455, 1997. 